\title{
Atomic Structure of Reduced Graphene Oxide
}

\author{
Cristina Gómez-Navarro, ${ }^{*}, \uparrow, \perp, \perp$ Jannik C. Meyer, ${ }^{\S, \perp}$ Ravi S. Sundaram, ${ }^{\dagger}$ Andrey Chuvilin, ${ }^{\S}$ \\ Simon Kurasch, ${ }^{\S}$ Marko Burghard, ${ }^{\dagger}$ Klaus Kern, ${ }^{\dagger,}{ }^{\prime \prime}$ and Ute Kaiser ${ }^{\S}$
}

\begin{abstract}
${ }^{\dagger}$ Max-Planck-Institut für Festkörperforschung, Heisenbergstrasse 1, 70569 Stuttgart, Germany, ${ }^{*}$ Departamento de Física de la Materia Condensada, Universidad Autónoma de Madrid, 28049 Madrid, Spain, ${ }^{\S}$ Electron Microscopy Group of Materials Sciences, University of Ulm, 89143, Germany, and "Ecole Polytechnique Fédérale de Lausanne, Institut de Physique de la Matière Condensée, 1015 Lausanne, Switzerland
\end{abstract}

\begin{abstract}
Using high resolution transmission electron microscopy, we identify the specific atomic scale features in chemically derived graphene monolayers that originate from the oxidation-reduction treatment of graphene. The layers are found to comprise defectfree graphene areas with sizes of a few nanometers interspersed with defect areas dominated by clustered pentagons and heptagons. Interestingly, all carbon atoms in these defective areas are bonded to three neighbors maintaining a planar $\mathrm{sp}^{2}$-configuration, which makes them undetectable by spectroscopic techniques. Furthermore, we observe that they introduce significant in-plane distortions and strain in the surrounding lattice.
\end{abstract}

KEYWORDS Graphene oxide, transmission electron microscopy, defects

S ince the discovery of graphene, ${ }^{1}$ a two-dimensional atomic sheet entirely made of carbon, immense efforts have been directed toward the development of reliable methods for obtaining large quantities of this extraordinary material. However, the so-far established approaches, most prominently the micromechanical cleavage of graphite, ${ }^{1}$ epitaxial growth on silicon carbide, ${ }^{2}$ chemical vapor deposition (CVD) of hydrocarbons on transition metal surfaces, ${ }^{3}$ as well as the dispersion of graphite in organic solvents, ${ }^{4}$ present difficulties in obtaining processable graphene sheets in large quantities thus impeding full exploitation of its exciting properties. One promising, low-cost, and easily up-scalable alternative is the reduction of graphite oxide, ${ }^{5-7}$ a layered material whose constituting graphene layers functionalized with epoxy and hydroxyl groups are easily exfoliated in water. The resulting graphene oxide (GO) monolayers can be deposited in controllable density onto a large variety of substrates, thus enabling the preparation of thin conductive films on solid and flexible substrates. ${ }^{8-10}$

Although chemical reduction of close-to-insulating GO can increase its conductivity by up to 4 orders of magnitude, ${ }^{1-13}$ thus far attained conductivities of reduced GO (RGO) still lack behind that of pristine graphene by a factor of $10-100 .^{14,15}$ There is general consensus that the inferior electrical performance of RGO originates from the presence of residual functional groups remaining after reduction. However, despite numerous spectroscopic ${ }^{16-19}$ and microscopic ${ }^{13,20,21}$ studies performed on both $\mathrm{GO}$ and RGO, their detailed atomic structure could not yet be determined due to their partial amorphous character. As a consequence, a variety

\footnotetext{
* To whom correspondence should be addressed. E-mail: cristina.gomez@uam.es

$\perp$ These authors contributed equally to this work.

Received for review: 09/24/2009

Published on Web: 03/03/2010
}

of structural models of GO are still debated. In the most popular model, GO is described as a random distribution of oxidized areas bearing the oxygenated functional groups, combined with nonoxidized regions wherein most of the carbon atoms preserve $\mathrm{sp}^{2}$ hybridization. ${ }^{18}$ Similar to other low-dimensional carbon nanostructures like carbon nanotubes $^{22}$ and fullerenes, ${ }^{23}$ topological defects in graphene are expected to strongly affect its electronic and mechanical properties, and thus to account for the differences between RGO and pristine graphene. A detailed study of the atomic structure of RGO hence represents a crucial step toward understanding the properties of this material. Here, we use high resolution transmission electron microscopy (TEM) to unravel the atomic structure of RGO monolayers. In contrast to mechanically exfoliated graphene, the chemically derived graphene is found to contain a considerable amount of topological defects, which could not be detected by previous spectroscopic and microscopic investigations.

Figure 1 a shows an optical micrograph of RGO-coated Quantifoil (QF) TEM grid with the coverage visible as grayish patches (see Supporting Information for further details on sample preparation). TEM imaging of the sample (Figure 1d) revealed that $\sim 1 \%$ of the holes of the grid were covered with sheets. Diffraction analysis was carried out to find holes covered by single layers. Single layers exhibited only one hexagonal pattern (Figure 1c) and the intensity ratios as described in ref 24. The occurrence of these hexagonal patterns implies a long-range orientational hexagonal order in the sheets, an observation not obvious from previous proposed models. A rapid search for single layers can be carried out by moving the sample with the objective lens defocused, such that a diffraction pattern is visible (in the image mode) when the beam is converged. In contrast to mechanically exfoliated graphene, the few-layer regions always exhibited multiple hexagonal patterns, implying that 


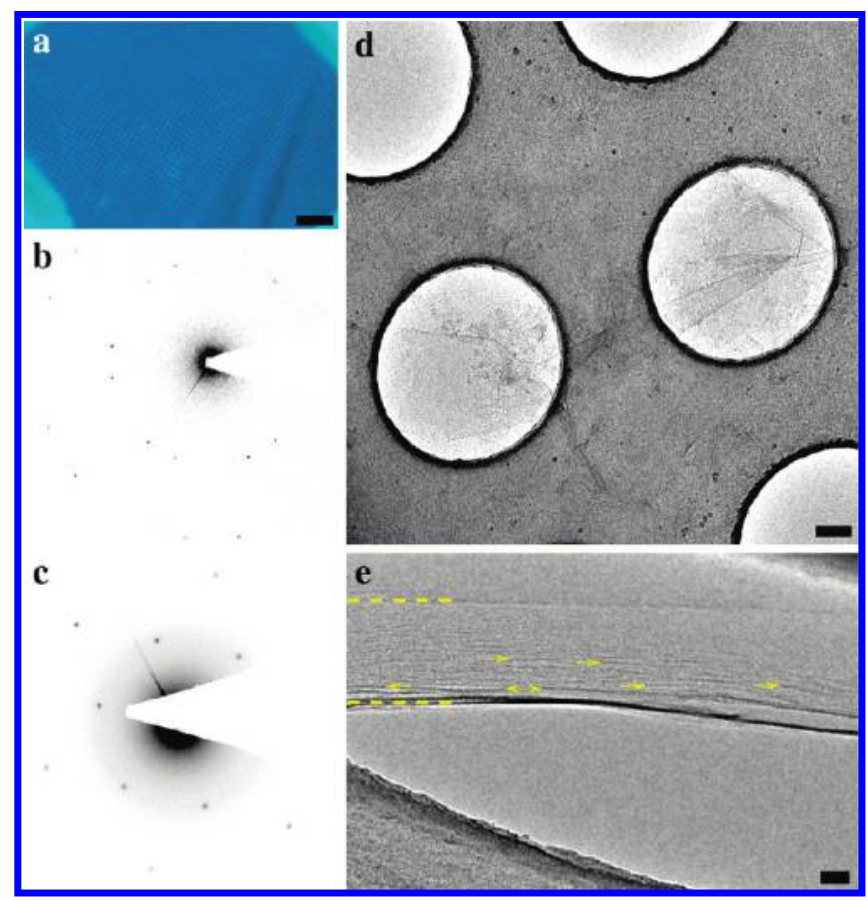

FIGURE 1. Single layer RGO membrane preparation and identification (a) Optical micrograph of graphene oxide sheets on the QF TEM grid. (b) Electron diffraction pattern of a bilayer area, showing the stacking with orientational mismatch of the sheets. (c) Diffraction pattern from a single layer. (d) TEM image of (partly folded) RGO sheets on the QF grid. (e) TEM image with the sample tilted to $60^{\circ}$. The region between the horizontal dashed lines is a single layer (region above is a double layer, below is vacuum). Arrows indicate horizontal dark lines where the RGO sheet appears parallel to the beam, indicating local deformations up to $30^{\circ}$. Scale bars are 10 $\mu \mathrm{m}(\mathrm{a}), 200 \mathrm{~nm}(\mathrm{~d})$, and $10 \mathrm{~nm}(\mathrm{e})$.

the multilayers are stacked in a turbostratic manner, unlike the AB Bernal stacking in graphite and mechanically exfoliated few-layer graphene. ${ }^{24,25}$ This turbostratic stacking is characteristic of low interacting sheets and corroborates full oxidation and exfoliation of the layers. Figure $1 b$ shows the parallel-beam diffraction pattern from a bilayer area in which two hexagonal patterns can be clearly observed. As a further observation, imaging the sample under a high tilt $\left(60^{\circ}\right.$, Figure $1 \mathrm{e}$ ) revealed a high roughness, much more than in mechanically exfoliated graphene. The horizontal dark lines within the sheet in Figure 1e (arrows) can be understood as the graphene layer parallel to the beam, locally in a deformed area. From this image alone, these wrinkles might be attributed to solution processing and drying, to stress, or to defects in RGO. However, as we show below, strong deformations in the membrane (observed as variations in the projected lattice spacing) are most prominent surrounding the extended defects in this material.

Comprehensive high resolution imaging of single layers provided insight into the exact atomic structure of the RGO layers, as exemplified by Figure 2. Different regions of the image are marked by colors in Figure $2 \mathrm{~b}$. It is apparent that the largest portion of the layer is comprised of clean wellcrystallized graphene areas where the hexagonal lattice is

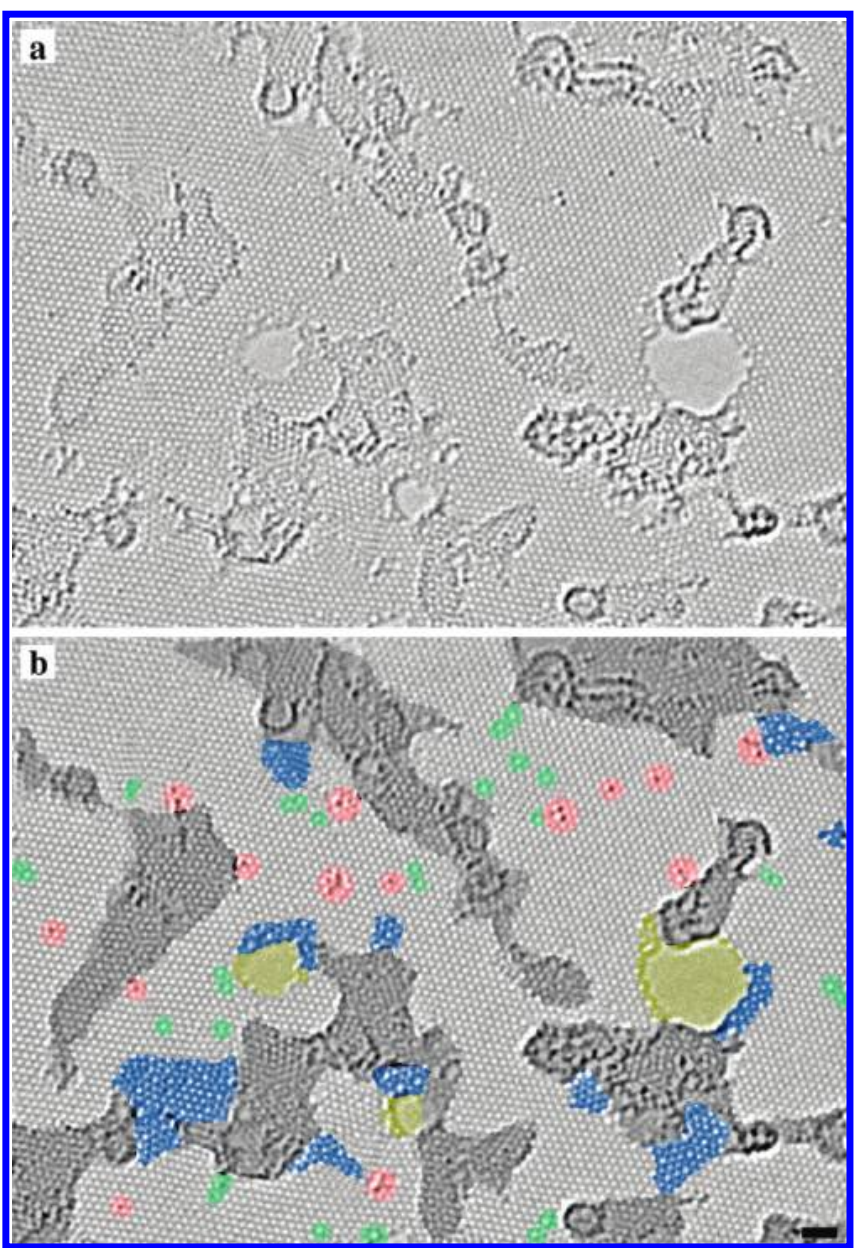

FIGURE 2. Atomic resolution, aberration-corrected TEM image of a single layer reduced-graphene oxide membrane. (a) Original image and (b) with color added to highlight the different features. The defect free crystalline graphene area is displayed in the original lightgray color. Contaminated regions are shaded in dark gray. Blue regions are the disordered single-layer carbon networks, or extended topological defects, that we identify as remnants of the oxidationreduction process. Red areas highlight individual ad-atoms or substitutions. Green areas indicate isolated topological defects, that is, single bond rotations or dislocation cores. Holes and their edge reconstructions are colored in yellow. Scale bar $1 \mathrm{~nm}$.

clearly observed (light gray color in Figure 2b). Akin to mechanically exfoliated graphene, we also observe a significant amount of regions where carbonaceous adsorbates and also heavier atoms are trapped ${ }^{26,27}$ (dark gray in Figure $2 b)$. The average size of the visible well-crystallized areas is from 3 to $6 \mathrm{~nm}$, and statistics reveal that they cover $60 \%$ of the surface. Another similarity to mechanically exfoliated graphene is the formation of larger holes under electron irradiation. However, in contrast to the latter, a significant amount of topological defects is observed within the clean areas. These can be further classified into isolated topological defects (pentagon-heptagon pairs, green), and extended (clustered) topological defects that appear as quasi-amorphous single layer carbon structures (marked in blue in Figure $2 \mathrm{~b}$ ). The extended topological defects cover ca. $5 \%$ of the surface and exhibit typical sizes of $1-2 \mathrm{~nm}$ in 
diameter. Unfortunately, it is impossible to determine the RGO membrane structure below the adsorbed contamination, which constitutes $\sim 30 \%$ of the total area. As the contaminations will preferably stick on defects, the portion of the defective regions is most likely underestimated. Surprisingly, despite the presence of such a significant amount of topological defects, the long-range orientational order is maintained. This can be discerned in the direct images, but is also clear from the diffraction patterns. It should be emphasized that the $80 \mathrm{keV}$ electron beam does not induce defects in pristine, crystalline graphene in any density that would be relevant for this study ${ }^{25}$ (although higher-energy electron irradiation indeed does induce similarlooking defects). Indeed, an annealing of the structure by relaxation of topological defects, rather than the creation of additional defects, seems to occur under prolonged $80 \mathrm{keV}$ irradiation (occasional bond rotations occur under the beam, which sometimes anneal a pentagon-heptagon pair but not an entire clustered area). Further, such defects were not observed in any mechanically exfoliated graphene samples. Indeed, some of our RGO and mechanically cleaved graphene samples were made from the same source graphite, so that it can be ruled out that the defects were already present in the source material. Thus, the high density of topological defects in the present images can be clearly attributed to graphene exfoliation by oxidation and reduction.

We now focus on these defect configurations and begin our discussion with the clustered topological defects that are marked in blue in Figure 2b, shown in Figure $3 a, b$, and encircled by dashed lines in Figure 4c,d. These extended defects predominantly incorporate a larger number of carbon pentagons, heptagons, and rotated hexagons in a nanometer-sized area. Nevertheless, all carbon atoms in these areas are bonded to three neighbors in a planar $\mathrm{sp}^{2}$. configuration. The absence of such defect configurations in mechanically exfoliated graphene identifies these clustered defects as a remnant of the oxidation-reduction process, which evolved from the originally strongly oxidized areas. While these clusters have not fully restored the crystalline, hexagonal graphene framework, they exhibit a planar geometry with a strong $\mathrm{sp}^{2}$-character. Their quasi-amorphous character results in disordered single-layer inclusions dominated by pentagons, hexagons, and heptagons within the membrane (Figure 3a,b and 4c,d). In Figure 3a, exemplary assignments of the most frequently occurring shapes, specifically carbon pentagons, hexagons, and heptagons, are made (a few octagons are also found). Using the intensity in the centers of these three polygons as a guide, it is then straightforward to discern the remaining structure in Figure 3a. Figure $3 b$ shows a partially assigned similar configuration, again consisting of multiple carbon polygons with no apparent order

The observed separation of the defective and crystalline areas in RGO is in agreement with the model proposed by Lerf and co-workers. ${ }^{18}$ This model gains further support by

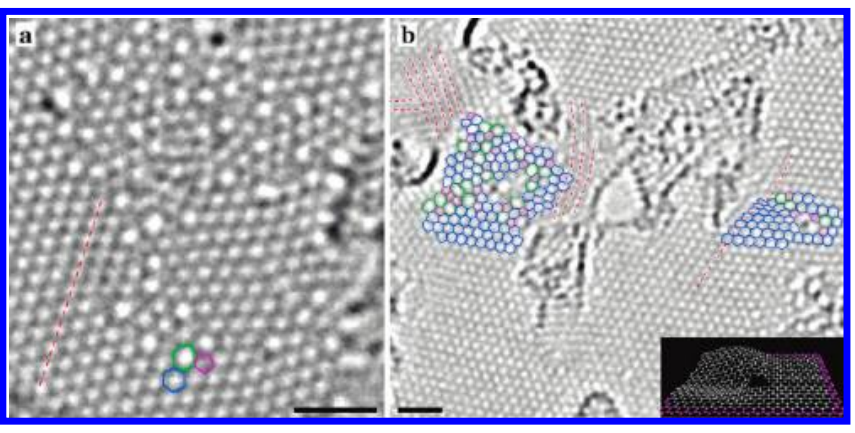

FIGURE 3. Extended topological defects and deformations in RGO. (a) Atomic resolution TEM image of a nonperiodic defect configuration. Dark contrast can be directly interpreted in terms of atomic structure. As examples, a carbon hexagon is indicated in blue, a pentagon in magenta, and a heptagon in green. (b) Partial assignment of the configurations in defective areas. Again, carbon pentagons, hexagons, and heptagons are indicated in magenta, blue, and green, respectively. The disordered carbon network is clearly visible. Further, red dashed lines indicate directions with strong deformations in the lattice. In addition to the curved shapes, the projected lattice period is reduced along some parts of the lines, indicating out-of-plane distortions. A local shortening of the projected period by $10 \%$ is often found, corresponding to $25^{\circ}$ local inclination of the membrane (while the defect-free regions are close to normal to the beam). This distortion is clearly seen along the example red dashed line in panel (a), where the lattice is shorter in the upper part. The structure on the right-hand side of panel (b) is a dislocation where an extra line of hexagons begins. For this example, a structural model similar to the observed configuration was generated and allowed to relax (inset), showing clearly the strong local deformations associated with this type of defect. The omitted central part of the defect was not stable during exposure. All scale bars are $1 \mathrm{~nm}$.

NMR studies ${ }^{17}$ which suggest that the majority of the carbons with hydroxyl and epoxide groups are arranged within the highly oxidized areas, along with undisturbed graphitic regions. While the exact atomic configurations in the oxidized state remain unclear, the present TEM images demonstrate that the oxidation-reduction process leaves disordered carbon inclusions within the sheets, which are seamlessly connected to the crystalline areas. Another relevant observation is that the graphene regions in vicinity to these defects can be highly distorted. For instance, in-plane distortions are seen as shifts in the direction of the lattice (red dashed lines in Figure 3b and 4c). Moreover, outof-plane distortions are observed as reduced projected lattice spacing in one direction. A reduction in the projected lattice of $10 \%$ (that is, from 2.46 to $2.2 \AA$ ) in one direction is often observed (cf. Figure 3a), reflecting a local inclination of the membrane of $\arccos (0.9)=25^{\circ}$. These distortions are typically limited to areas immediately adjacent to the defect clusters, while the larger defect-free graphene areas appear undistorted.

We now turn to the isolated topological defects, which provide indirect evidence that the RGO sheets have been severely strained at some point of the process and are still under a significant strain. Most of the isolated topological defects in Figure 2 (marked in green in Figure 2b) are dislocation cores that incorporate a pentagon-heptagon pair. The presence of such defects is usually the result of a 


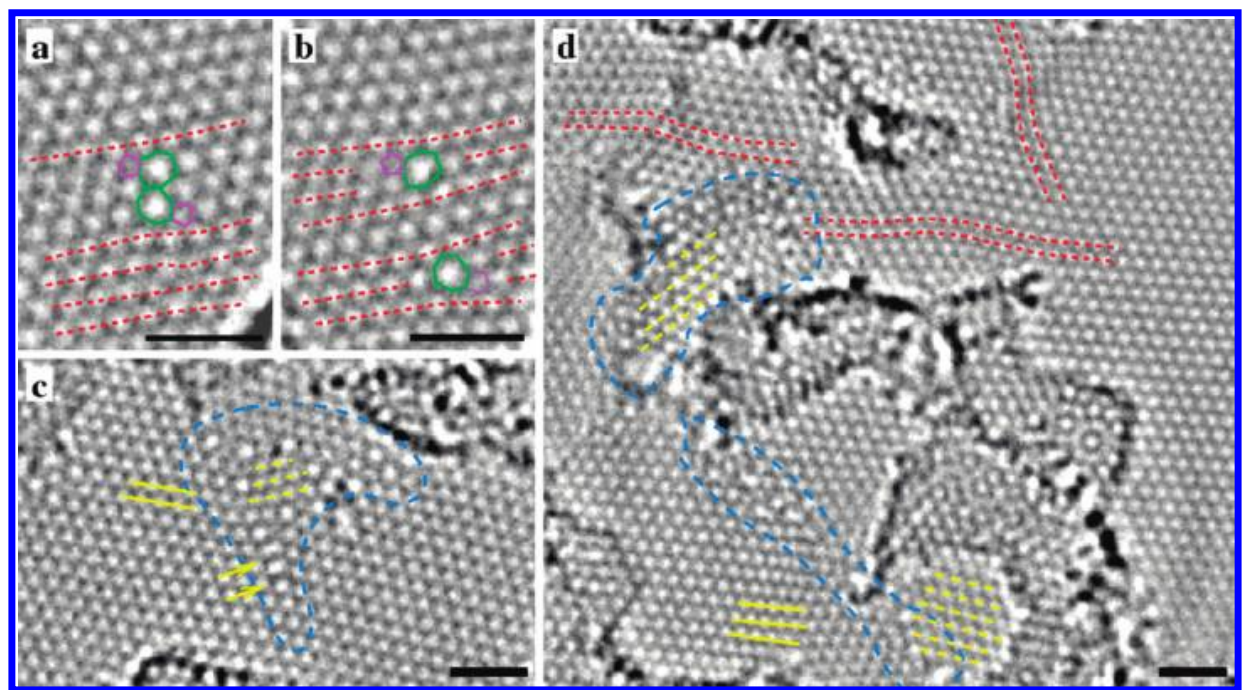

FIGURE 4. (a,b) Dislocation dipole observed at two different times ( 2 min apart) in the TEM. Each dislocation core consists of a pentagon-heptagon pair. The separation between the dislocation cores has increased, indicating that there is a significant amount of strain present. (c,d) Defect clusters, indicated by blue dashed lines. Yellow dashed lines indicate regions with a hexagonal lattice rotated to the dominant orientation (yellow solid lines for comparison) is observed. Red dashed lines indicate again distortions in the hexagonal lattice. Yellow arrows in (c) indicate strongly elongated carbon polygons. All scale bars are $1 \mathrm{~nm}$.

plastic deformation. Figure 4a,b shows a dislocation dipole, comprising two pentagon-heptagon pairs, at two different time points under continuous observation in the TEM. The pentagon-heptagon pairs separate, thereby extending the dislocation dipole in the process. The activation energy required to overcome a migration barrier for the defect might be provided by the energy input from the electron beam. However, the fact that the pentagon-heptagon pairs separate (rather than anneal, as observed in crystalline graphene) ${ }^{25}$ indicates that there still is sufficient strain to favor the separation. One can even find highly elongated carbon polygons, as indicated by arrows in Figure 4c.

A few more observations in connection with these defects are worth noting. Figure 4d shows two larger defect clusters, indicated by the blue dashed lines. For one of them, the defects are clustered along a line, reminiscent of a grain boundary (this observation agrees with the predictions of Car and co-workers). ${ }^{28}$ In close proximity, one observes small patches of hexagonal lattice (typically $1 \mathrm{~nm}$ across), which are rotated with respect to the dominant graphene orientation (yellow dashed lines in Figure 4c,d). Again, these are likely to originate from the oxidation-reduction process, albeit in this case the reduction/annealing created a small hexagonal (graphene-like) area within the defect. We note that such a configuration might be most difficult to anneal, since it is already in the optimum (hexagonal) configuration locally, but mismatched to the dominant lattice orientation.

In summary, we have presented a real space atomic resolution study of the structure of RGO. The TEM data reveal that the sheets are composed of intact graphene islands of variable size between 3 and $6 \mathrm{~nm}$, interspersed with defect clusters forming planar, quasi-amorphous $\mathrm{sp}^{2}$-bonded areas. These findings are in accord with the model proposed by Lerf and co-workers, ${ }^{17,18}$ but also reveal the presence of a remarkable amount of topological defects after reduction. Overall, the following scenario emerges for the oxidationreduction process in GO: Upon oxidation, isolated highly oxidized areas (few $\mathrm{nm}$ in size) are formed while at least ca. $60 \%$ of the surface remains undisturbed. Upon reduction, the oxidized areas are restored to $\mathrm{sp}^{2}$-bonded carbon networks, which however lack the perfect crystallinity of intact graphene. The reduced disordered areas, which are best described as clustered topological defects, induce strain as well as in-plane and out-of-plane deformations in the surrounding RGO. Isolated topological defects, mostly dislocations, are also present and may have formed as a result of strain. The effects of these defects will have to be taken into account for any comprehensive study of the properties of this material. Finally knowledge of the defect structures may help to devise procedures to remove them or alternatively, may lead to novel applications that take specific advantage of their presence.

Supporting Information Available. A detailed description of the sample preparation and experimental methods. This material is available free of charge via the Internet at http:// pubs.acs.org.

\section{REFERENCES AND NOTES}

(1) Novoselov, K. S.; Jiang, D.; Schedin, F.; Booth, T. J.; Khotkevich, V. V.; Morozov, S. V.; Geim, A. K. Proc. Natl. Acad. Sci. U.S.A. 2005, $102(30), 10451-10453$

(2) Berger, C.; Song, Z. M.; Li, T. B.; Li, X. B.; Ogbazghi, A. Y.; Feng, R.; Dai, Z. T.; Marchenkov, A. N.; Conrad, E. H.; First, P. N.; de Heer, W. A. I. Phys. Chem. B 2004, 108 (52), 19912-19916.

(3) Kim, K. S.; Zhao, Y.; Jang, H.; Lee, S. Y.; Kim, J. M.; Ahn, J. H.; Kim, P.; Choi, J. Y.; Hong, B. H. Nature 2009, 457 (7230), 706710 .

(4) Hernandez, Y.; Nicolosi, V.; Lotya, M.; Blighe, F. M.; Sun, Z. Y.; De, S.; McGovern, I. T.; Holland, B.; Byrne, M.; Gun'ko, Y. K.; Boland, J. J.; Niraj, P.; Duesberg, G.; Krishnamurthy, S.; Goodhue, 
R.; Hutchison, J.; Scardaci, V.; Ferrari, A. C.; Coleman, J. N. Nat. Nanotechnol. 2008, 3 (9), 563-568.

(5) Park, S.; Ruoff, R. S. Nat. Nanotechnol. 2009, 4 (4), 217-224.

(6) Stankovich, S.; Dikin, D. A.; Dommett, G. H. B.; Kohlhaas, K. M.; Zimney, E. J.; Stach, E. A.; Piner, R. D.; Nguyen, S. T.; Ruoff, R. S Nature 2006, 442 (7100), 282-286

(7) Stankovich, S.; Piner, R. D.; Chen, X. Q.; Wu, N. Q.; Nguyen, S. T.; Ruoff, R. S. I. Mater. Chem. 2006, 16 (2), 155-158.

(8) Wang, X.; Zhi, L. J.; Mullen, K. Nano Lett. 2008, 8 (1), 323-327.

(9) Eda, G.; Fanchini, G.; Chhowalla, M. Nat. Nanotechnol. 2008, 3 (5), 270-274.

(10) Li, D.; Muller, M. B.; Gilje, S.; Kaner, R. B.; Wallace, G. G. Nat. Nanotechnol. 2008, 3 (2), 101-105

(11) Jung, I.; Dikin, D. A.; Piner, R. D.; Ruoff, R. S. Nano Lett. 2008, 8 (12), 4283-4287.

(12) Gilje, S.; Han, S.; Minsheng, W.; Kang, L. W.; Kaner, R. B. Nano Lett. 2007, 7 (11), 3394-3398.

(13) Gomez-Navarro, C.; Weitz, R. T.; Bittner, A. M.; Scolari, M.; Mews, A.; Burghard, M.; Kern, K. Nano Lett. 2007, 7 (11), 3499-3503.

(14) López, V.; Sundaram, R. S.; Gómez-Navarro, C.; Olea, D.; Burghard M.; Gómez-Herrero, J.; Zamora, F.; Kern, K. Adv. Mater. 2009, 21 (46), 4683-4687.

(15) Tung, V. C.; Allen, M. J.; Yang, Y.; Kaner, R. B. Nat. Nanotechnol 2009, 4 (1), 25-29.

(16) Szabo, T.; Berkesi, O.; Forgo, P.; Josepovits, K.; Sanakis, Y.; Petridis, D.; Dekany, I. Chem. Mater. 2006, 18 (11), 2740-2749.

(17) Cai, W. W.; Piner, R. D.; Stadermann, F. J.; Park, S.; Shaibat, M. A.; Ishii, Y.; Yang, D. X.; Velamakanni, A.; An, S. J.; Stoller, M.; An,
J. H.; Chen, D. M.; Ruoff, R. S. Science 2008, 321 (5897), 1815 1817 .

(18) Lerf, A.; He, H. Y.; Forster, M.; Klinowski, J. L.Phys. Chem. B 1998, 102 (23), 4477-4482.

(19) Kudin, K. N.; Ozbas, B.; Schniepp, H. C.; Prud'homme, R. K.; Aksay, I. A.; Car, R. Nano Lett. 2008, 8 (1), 36-41.

(20) Pandey, D.; Reifenberger, R.; Piner, R. Surf. Sci. 2008, 602 (9), $1607-1613$.

(21) Mkhoyan, K. A.; Contryman, A. W.; Silcox, J.; Stewart, D. A.; Eda, G.; Mattevi, C.; Miller, S.; Chhowalla, M. Nano Lett. 2009, 9 (3), $1058-1063$.

(22) Gomez-Navarro, C.; Pablo, P. J. D.; Gomez-Herrero, J.; Biel, B.; Garcia-Vidal, F. J.; Rubio, A.; Flores, F. Nat. Mater. 2005, 4 (7), 534.

(23) Saito, R.; Dresselhaus, G.; Dresselhaus, M. S. Chem. Phvs. Lett. 1992, 195 (5-6), 537-542.

(24) Meyer, J. C.; Geim, A. K.; Katsnelson, M. I.; Novoselov, K. S.; Booth, T. J.; Roth, S. Nature 2007, 446 (7131), 60-63.

(25) Meyer, J. C.; Kisielowski, C.; Erni, R.; Rossell, M. D.; Crommie, M. F.; Zettl, A. Nano Lett. 2008, 8 (11), 3582-3586.

(26) Gass, M. H.; Bangert, U.; Bleloch, A. L.; Wang, P.; Nair, R. R.; Geim, A. K. Nat. Nanotechnol. 2008, 3 (11), 676-681.

(27) Meyer, J. C.; Girit, C. O.; Crommie, M. F.; Zettl, A. Nature 2008, 454 (7202), 319-322.

(28) Li, J. L.; Kudin, K. N.; McAllister, M. J.; Prud'homme, R. K.; Aksay, I. A.; Car, R. Phys. Rev. Lett. 2006, 96, (17), 176101 